\title{
Juan V. Martín Applications of a New Property of Conics to Zorraquino Architecture: \\ Francesco \\ Granero An Alternative Design Project for Rio de Janeiro Metropolitan Cathedral
}

Rodríguez

José Luis Cano

This paper describes the mathematical discovery of a new property of conics which allows the development of numerous geometric projects for use in architectural and engineering applications. Illustrated is an architectural application in the form of an alternative project for Río de Janeiro Metropolitan Cathedral featuring the integration of a circular base and a cross in the top plane. Two alternative designs are presented for the cathedral, based on the choice of either the Latin Immisa or Greek cross.

\section{Applications of a new property of conics in Architecture}

In 1993 we set forth a new property of conics [Martín-Zorraquino, et al. 1993]. Subsequent papers presented at three international conferences (ISAMA 99, the 9th International Congress of Graphic Engineering and Mathematics and Design 98) examined some interesting examples of the applications of this new property of conics in architecture and in engineering [MartínZorraquino, et al. 1999; Martín-Zorraquino, et al. 1998; Martín-Zorraquino, et al. 1997]. This paper sets forth the architectural possibilities that a new mathematical discovery allows us to achieve. First, the new property of conics has been proved, along with their two particular cases, further analysing a family of generated ellipses $\mathrm{E}(\mathrm{M})$ in such a way that by means of a simple law of linear arrangement, it is possible to generate the three-dimensional surface such that the sections perpendicular to the Z-axis are ellipses, proof of their ruled character. Next, we take as our example two alternative arrangements for Rio de Janeiro Metropolitan cathedral, setting forth the interpenetration and parametrization techniques carried out in order to achieve these geometrical projects. In each arrangement a circular base is linked to a cross on the ridge above and parallel to the plane where the base circumference is located. These arrangements have the novel feature of the cross as an integral part of the construction, and the difference between the two possibilities depends on the style of cross chosen, that is, whether the Latin Immisa or the Greek cross is preferred. The three-dimensional development of the project has been achieved by the development of computer programs that have enabled us to depict the two arrangements in three-space, while working out an interesting method of parametrization of the final conic surface as a result of the process of interpenetration of two or more surfaces. Finally, we deal with aspects of construction both with concrete and metallic structures for the attainment of the geometric projects, such as, the original construction techniques for concrete shells used widely by Spanish arquitect Félix Candela in countries such as México, Colombia, the United States and Spain.

\section{Mathematical Demonstration of the new property of conics particularized to the ellipse} case

In the projects set out here as alternatives to the actual Rio de Janeiro Metropolitan Cathedral, we have made use of the new property of conics [Martin-Zorraquino et al, 1993] adapted to the particular case of the ellipse. Hence, all the mathematical calculus needed for the graphic design 
of the projects are based on the expressions that result from of the demonstration of the new property of conics for the case of the ellipse, as this philosophy of calculation can be extended, as is described below, to the other two remaining conics, namely, the hyperbola and the parabola.

Beginning then, with the demonstation of the new property of conics, let us first consider ellipse $M$ (Figure 1), defined in the plane $u v$ by the equation for the ellipse [see Appendix I]:

$$
\frac{u^{2}}{a^{2}}+\frac{v^{2}}{b^{2}}=1, a \neq b
$$

where $a$ stands for the major semiaxis of the ellipse and $b$ the minor semiaxis of the ellipse.

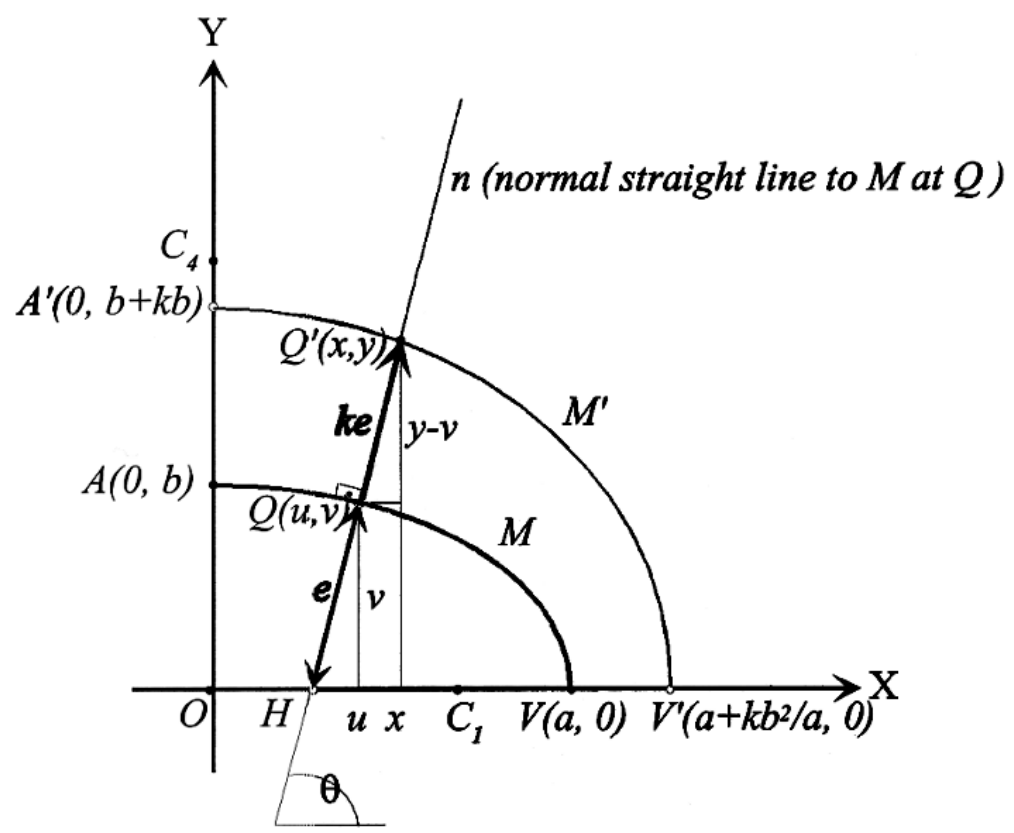

Figure 1

A straight line normal to ellipse $M$ at point $Q$ has been drawn, showing only a quarter of the ellipse, as in Figure 1, from where the following property shall be demonstrated:

The loci of the points $(x, y)$ mirrors of points $(u, v) \in M$, through the mapping stated in this figure $\left(k \in \mathfrak{R}\right.$ ), is in its turn another ellipse $M^{\prime}$. The exception point $V$ (apex of $M$ ) together with its image point $V^{\prime}$ (apex of $M^{\prime}$ ), yields to:

$$
V V^{\prime}=k \frac{b^{2}}{a}
$$

where the value $b^{2} / a$ corresponds to the curvature radius at point $V(a, 0)$, being represented in the figure by the distance from point $C_{1}$ to $V(a, 0)$. In the family $E(M)$ of ellipses generated for

44 NEXUS NETWORK JOURNAL - VOL. 3, NO. 1, 2001 
the values $k \in \mathfrak{R}$, there are two circumferences and two straight lines (ellipses with a null semiaxis).

In order to prove this property of the ellipse, which is common to all three conics, as further demonstrated, let us first arrive at the slope of the straight line $n$ normal to the generator ellipse $M$ at point $Q(u, v)$.

Deriving the equation (1) it yields:

$$
v^{\prime}=-\frac{b^{2} u}{a^{2} v}
$$

Hence, the slope of the straight line normal to the tangent straight line $Q(u, v)$ is yielded through the condition of perpendicularity between the two straight lines: $m m^{\prime}=-1$.

Thus, it results that $m^{\prime}=-1 / m$, and therefore:

$$
\operatorname{tg} \theta=\frac{a^{2} v}{b^{2} u}
$$

is the slope being sought.

Due to the similarities between triangles, $\frac{v}{e}=\frac{y-v}{k e} \Rightarrow v=\frac{1}{k+1} y$

(but the reader should note that $\forall Q \neq V, e \neq 0$ ).

Again, referring to Figure 1, making calculations:

$$
\operatorname{tg} \theta=\frac{a^{2} v}{b^{2} u}=\frac{y-v}{x-u}=\frac{(k+1) v-v}{x-u} \Rightarrow \frac{a^{2}}{b^{2} u}=\frac{k}{x-u}, v \neq 0
$$

yields:

$$
u=\frac{a^{2} v}{\left(a^{2}+k b^{2} v\right)^{x}}
$$

and since $Q \neq V(V \neq 0)$, it brings about:

$$
u=\frac{a^{2}}{a^{2}+k b^{2}} x
$$

By substituting the previous expressions of $u, v$ into equation (1), eventually it turns out the following points loci $Q^{\prime}(x, y)$ :

$$
E(M) \equiv \frac{x^{2}}{\left(\frac{a^{2}+k b^{2}}{a}\right)^{2}}+\frac{y^{2}}{[b(1+k)]^{2}}=1
$$


This represents the Cartesian equation of an ellipse for all $k \in \mathfrak{R}$, and by depending on the parameter $k$, it constitutes the equation of a family of ellipses $E(M)$, generated from the generator ellipse $M$ defined according to the equation (1).

\section{Analysis of the ellipse family $\mathrm{E}(\mathrm{M})$}

In the ellipse family $E(M)$ generated from the generator ellipse $M$ defined according to equation (1), there are two circumferences and two straight lines, which is further demostrated in Figure 2, where such particular cases have only been represented in one quarter of the two-dimension representation.

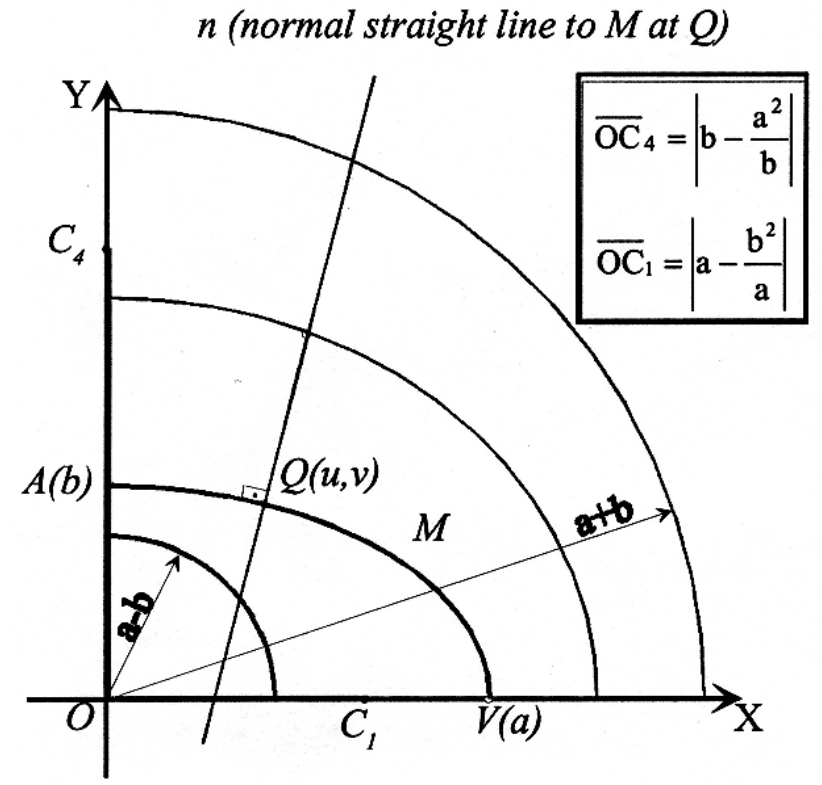

Figure 2

Identifying the semiaxis of $E(M)$, we have

$$
\frac{a^{2}+k b^{2}}{a}= \pm(k+1) b \Rightarrow\left\{\begin{array}{l}
k=-\frac{a}{b} \\
k=\frac{a}{b}
\end{array}\right.
$$

Substituting those values into equation (6) gives rise respectively to the circumferences:

$$
\begin{aligned}
& x^{2}+y^{2}=(a+b)^{2} \\
& x^{2}+y^{2}=(a-b)^{2}
\end{aligned}
$$

In the same way, by making each of the semiaxes equal to zero yields: 


$$
\begin{array}{lll}
(k+1) b=0 & \Rightarrow & k=-1 \\
\frac{a^{2}+k b^{2}}{a}=0 & \Rightarrow & k=-\frac{a^{2}}{b^{2}}
\end{array}
$$

The equality $k=-1$ gives rise to a symmetrical and horizontal segment whose center matches the point $O$ (the origin of the coordinates), with $C_{1}$ as one of its edges (Figures 2 and 3).

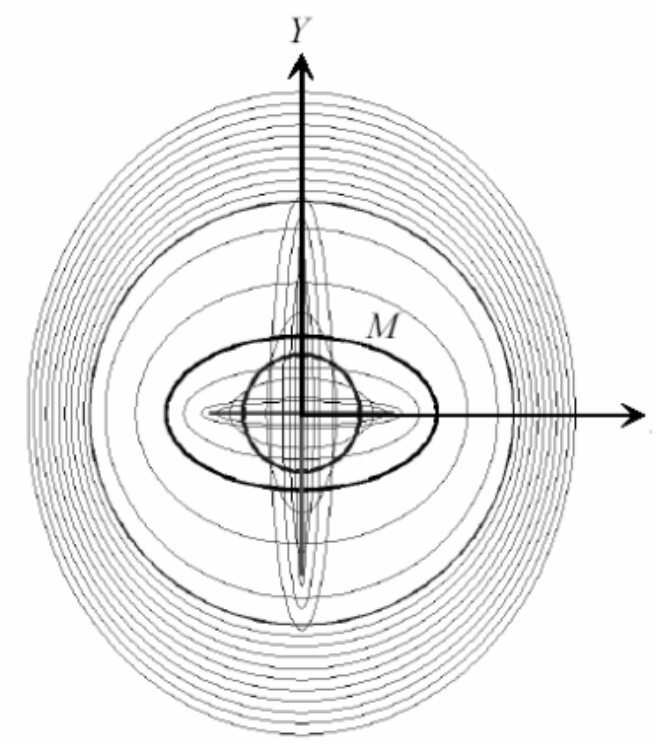

Figure 3

With $k=a^{2} / b^{2}$, we have a symmetrical and vertical segment with its center is the point $\mathrm{O}$, and $C_{4}$ as one of its edges.

It can be easily proven that $\left|a-b^{2} / a\right| y\left|b-a^{2} / b\right|$, are respectively the semilengths of such segments, noting the fact that the segment $\overline{C_{1} C_{2}}$ represents the distance between the curvature centers in the horizontal axis; in the same way the segment $\overline{C_{3} C_{4}}$ represents the distance between the curvature centers in the vertical axis .

Figure 3 is a computer representation of different values of $k$, two straight lines, two circumferences and several ellipses, among which can be noticed the generator ellipse $M$ of the family.

Every generated point and generated conic remains defined in their totality by a numerical parameter $k$ referred to as "the generator conic". This constitutes a thoroughly exclusive property in comparison to any other known method of generating surfaces with conics.

In the process of generating conics of the family $E(M)$, we can find semi-straight lines or straight lines (depending on whether the generator conic is an ellipse, hyperbola or parabola). 
Thus, a straight line can be dealt with mathematically and geometrically as a conic belonging to the family.

In the case of the generator ellipse, two circumferences are obtained that can be handled as conics of the family.

As a corollary, the following can be stated:

- A new concept appears: that of the "generator ellipse", or in general, the "generator conic" belonging to the corresponding family of generated conics.

- It is a novelty to handle the straight lines as ellipses with a null semi-axis.

\section{Generation of surfaces from the family $\mathrm{E}(\mathrm{M})$}

If it were possible to arrange in space, in a continuous way, this entire family of generated conics, we would obtain a surface having cross-sections that would also belong to the family. This guideline for arranging the ellipses will be called the "Arrangement Law" and shall correspond to a "generating line" upon which all transformed points coming from a single point of the generator ellipse shall rest. This means that each $k$ applied to the generator ellipse will have its corresponding point upon the generating line in space. That is to say, each $k$ applied to the generator ellipse will have its corresponding ellipse in space, which is an additional arrangement condition for all parallel planes.

Each point belonging to the generator conic creates lines of the same family according to the Arrangement Law. So, if this Arrangement Law is a linear function of or proportional to the generating factor $k$, those lines shall be straight lines belonging to a ruled surface.

It is especially important to mention here in reference to matters of construction that those points belonging to the generating lines can be defined at once, by simply linking the homologous points between a straight line segment and a circumference. Thus a whole set of ellipses are obtained without the need to resort to traditional means for their generation. The simplicity of this mathematical treatment deserves note as a remarkable property, in contrast to the fact that usually the only simple treatment of similar ruled surfaces in science and technology is graphic. It is outstanding that, thanks to the Arrangement Law, an infinity of surfaces, whether linear or not, can be settled up.

Substituting $k$ for $z$ in equation (6) according to the linear relation $k=c z$, for $c=1$, we have:

$$
E(M) \equiv \frac{x^{2}}{\left(a+\frac{b^{2}}{a} z\right)^{2}}+\frac{y^{2}}{[b(1+z)]^{2}}=1
$$

which is the equation for a surface having cross-section curves that are the ellipses of the family $E(M)$, the $z$-coordinate being null in the generator ellipse plane, where all director curves or cross-section curves are located in parallel planes and perpendicular to the vertical axis $Z$.

Although the study of the surface with this equation was first undertaken with the aim of representing or characterizing the corresponding mathematical model, it has given rise up to the ascertainment of remarkable properties and applications, especially in construction, owing largely 
to the fact that these surfaces are ruled ones, as shall be further demonstrated. In Figure $4, k=z / c$ has been chosen according to the linear Arrangement Law.

\section{Confirmation of the condition of ruled surfaces}

In order to prove this important characteristic of our surfaces defined according to equation (7), its Cartesian equation shall have to be expressed through its parametric form:

$$
\left\{\begin{array}{l}
\mathrm{x}=\mathrm{m}(\zeta) \mathrm{z}+\mathrm{s}(\zeta) \\
\mathrm{y}=\mathrm{n}(\zeta) \mathrm{z}+\mathrm{q}(\zeta)
\end{array}\right.
$$

as demonstrated in Appendix III.

Establishing the relation between the equation:

$$
\frac{x^{2}}{\left(a+\frac{b^{2}}{a} z\right)^{2}}+\frac{y^{2}}{[b(1+z)]^{2}}=1
$$

and the fundamental trigonometric form: $\sin ^{2} \zeta+\cos ^{2} \zeta=1$, it is evident that:

$$
\left.\begin{array}{c}
\sin \zeta=\frac{\mathrm{x}}{\mathrm{a}+\frac{\mathrm{b}^{2}}{\mathrm{a}} \mathrm{z}} \\
\cos \zeta=\frac{\mathrm{y}}{\mathrm{b}(1+\mathrm{z})}
\end{array}\right\} \Rightarrow\left\{\begin{array}{ll}
\mathrm{x}=\frac{\mathrm{b}^{2}}{\mathrm{a}} \sin \zeta & \mathrm{z}+\mathrm{a} \sin \zeta \\
\mathrm{y}=\mathrm{b} \cos \zeta & \mathrm{z}+\mathrm{b} \cos \zeta
\end{array}\right\}
$$

and, identifying the equations (8) and (9), it can be proved that the paramenter $\zeta$ can be easily removed to lead to equation (7), quod erat demostrandum.

\section{Example of using the Arrangement Law}

As has been pointed out in the preceding section, depending on the relationship established between the parameter $k$ that defines the set of cross-section curves belonging to the family of ellipses and the variable $z$, an infinity of surfaces will result. A special case of these surfaces occurs when the relationship between $z$ and $k$ is linear, resulting in a ruled surface, as was previously demonstrated. This problem could be resolved by finding the equation of the ruled surface and its generating straight lines in order to develop a cover between a straight ridge and an elliptic base, given:

- Semimajor axis $A$ and semiminor axis $B$ of the elliptic base. 


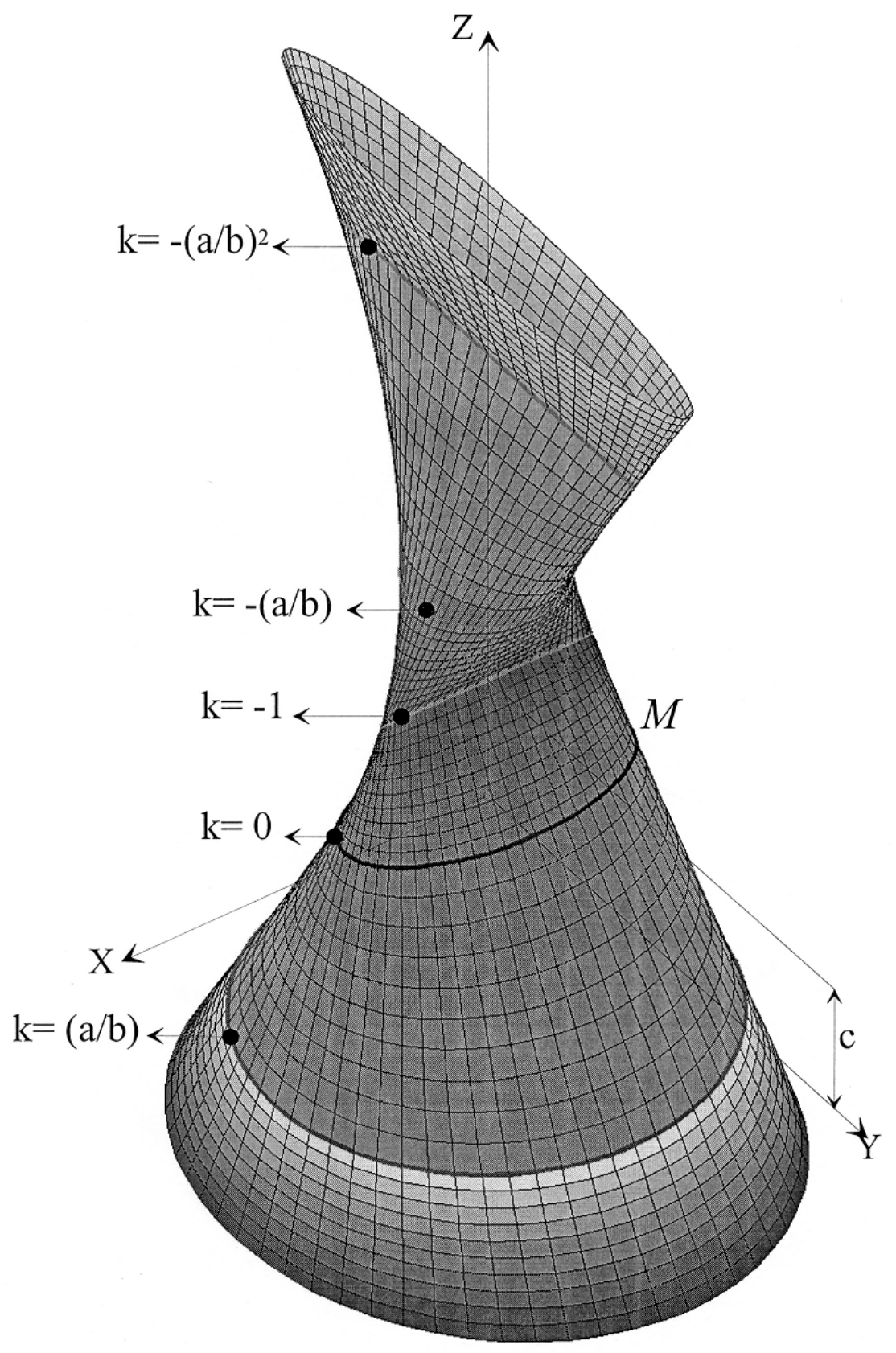

Figure 4

50 NEXUS NETWORK JOURNAL - VOL. 3, NO. 1, 2001 
- Height $H$ of the straight ridge.

- Length $L$ of the straight ridge (aligned with $A$ ).

The general equation of all generated ellipses by the generator ellipse with semiaxes $a$ and $b$ will be as already stated, according to equation (6):

$$
\frac{\mathrm{x}^{2}}{\left(\frac{\mathrm{a}^{2}+\mathrm{kb} \mathrm{b}^{2}}{\mathrm{a}}\right)^{2}}+\frac{\mathrm{y}^{2}}{[\mathrm{~b}(1+k)]^{2}}=1
$$

In the particular case for the base:

$$
\begin{aligned}
& A=\frac{a^{2}+k b^{2}}{a} \\
& B=b(1+k) \quad \Rightarrow \quad k o=\frac{B-b}{b}
\end{aligned}
$$

For the straight ridge, the corresponding ellipse would have semiaxis $L / 2$ and 0 , for $k=-1$

$$
\frac{L}{2}=a-\frac{b^{2}}{a} \Rightarrow b^{2}=a\left(a-\frac{L}{2}\right)
$$

taking the center of the generator ellipse as the origin of the coordinates, and considering it located between the straight ridge and the base at a distance $c$ below the straight ridge. The linear relation between $k$ and $z$ can be set forth when it is known that in the straight ridge $z=c$ for $k=$ -1 , and in the generator ellipse $z=0$ for $k=0$ :

$$
k=-\frac{z}{c}
$$

which for the coordinate

$$
z=c-H \Rightarrow k=-\frac{c-H}{c}
$$

From (11) and (13),

$$
k=\frac{A-a}{a-\frac{L}{2}}
$$

From (12) and (15),

$$
b=\frac{B(a-L / 2)}{A-L / 2}
$$


From (13) and (16),

$$
a=\frac{B^{2} \frac{L}{2}}{B^{2}-(A-L / 2)^{2}}
$$

From (14) and (15),

$$
c=\frac{H}{\frac{A-a}{a-L / 2}+1}
$$

With values $a, b$ and $c$ the resulting surface equation $\mathrm{f}(x, y, z)=1$ is:

$$
\frac{x^{2}}{\left(\frac{a^{2}-(z / c) b^{2}}{a}\right)}+\frac{y^{2}}{\left[b\left(1-\frac{z}{c}\right)\right]^{2}}=1
$$

Another interesting value could be the distance $I$ between the two straight lines (ellipses with a null semiaxis):

$$
I=\frac{c a^{2}}{b^{2}}-c
$$

The two circumferences answer to the condition that their semiaxes $A$ and $B$ are equal $(A=B)$, for values of $k$ such that $k=-a / b$ and $k=a / b$. All of what has been calculated is shown in Figure 4.

In order to obtain the straight lines (generating lines according to the concept of ruled surfaces), it is sufficient to take several points of the generator ellipse and apply to them the values $k=-1$ (straight ridge) and $k=k$ (base circumference). First, we shall have to obtain the equation of the normal straight line at the selected point of the generator ellipse. Second, we shall draw the intersection between the normal straight line previously traced and the transformed ellipses $(k=-1$ and $k=k \mathrm{o})$, obtaining in this way the coordinates $x, y$ of the end of the straight line. The coordinate $z$ stands for the distance with respect to the generator ellipse.

\section{Geometrical and Mathematical Development of the Project}

The project of the alternate version of the Rio de Janeiro Cathedral in Brasil has been developed as an example of a direct application of the new property of conics to architecture, with the particular requirement that the design shall include a cross at its straight ridge as an integral part of such a construction. Two patterns of crosses have been set out in this paper, the Latin Immisa cross and the Greek cross. The Greek cross has two arms of equal length that cross each other at right angles, the point of intersection being at the center points of the arms. The Latin Immisa cross has arms of unequal length that cross each other at right angles; the point of intersection, as in the Greek cross, is at the center points of the arms. This is different from the more common Latin cross, in which the point of intersection does not coincide with the midpoint of the long arm.

52 NEXUS NETWORK JOURNAL - VOL. 3, NO. 1, 2001 


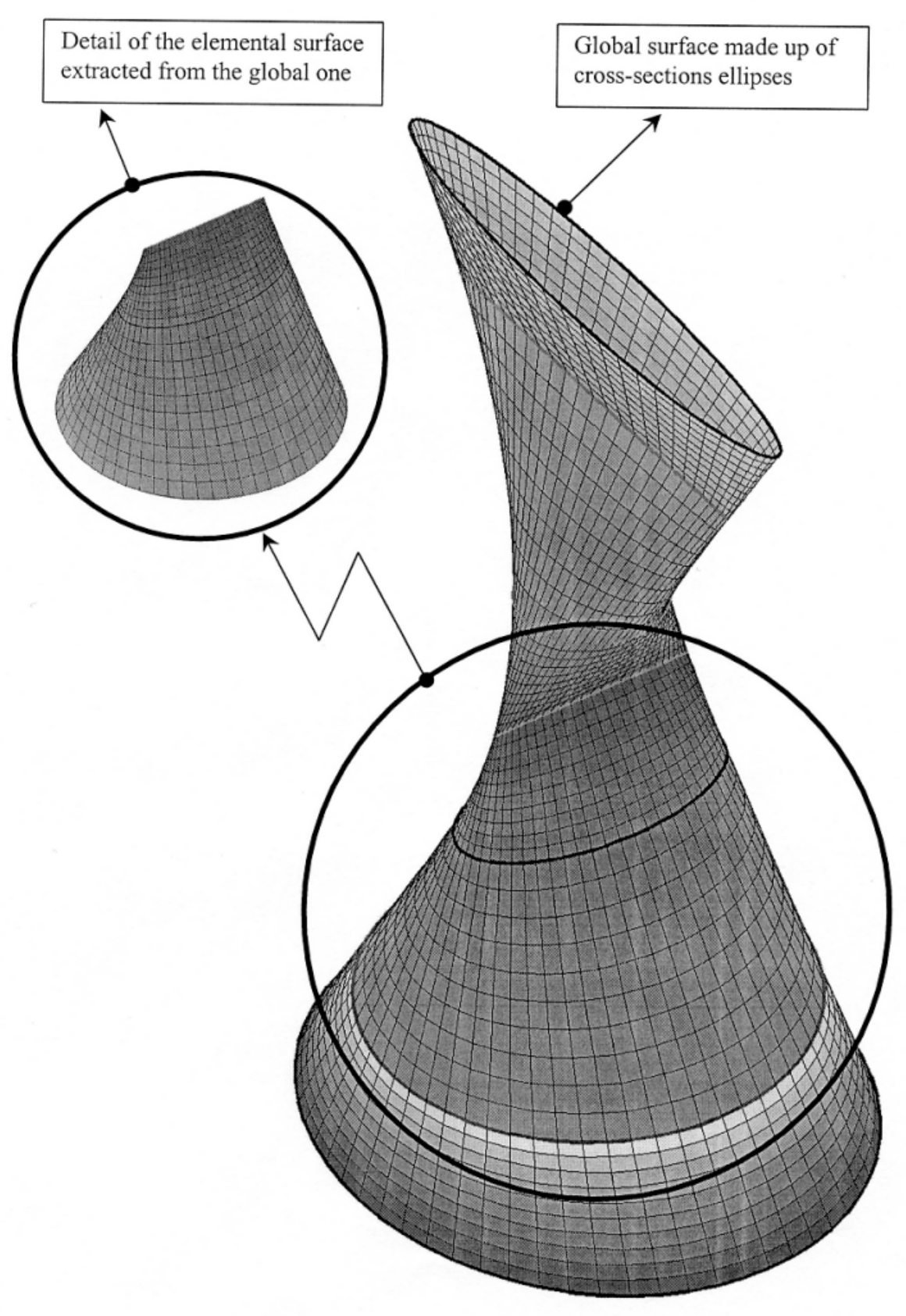

Figure 5

NEXUS NETWORK JOURNAL - VOL. 3, NO. 1, 200153 
The fact that each cross is composed of two arms perpendicular to each other, irrespective of their lengths, made us think about the intersection of two surfaces having straight ridges that were rotated by $90^{\circ}$. On the other hand, since the base to be linked with the straight ridge consists of a circumference (in this particular case, of an ellipse), the only possibility we have, judging by all that has been dealt with in the preceding sections, is the employment of the family of ellipses $E(M)$ expressed by by equation (6), which is repeated here:

$$
E(M) \equiv \frac{x^{2}}{\left(\frac{a^{2}+k b^{2}}{a}\right)^{2}}+\frac{y^{2}}{[(1+k) b]^{2}}=1
$$

Endowing the former expression with a linear Arrangement Law of the form $k=-z / c$, where $c$ represents the height between the ridge plane and the plane of the generator ellipse, we obtain the following expression both in Cartesian and parametric forms:

$$
\frac{x^{2}}{\left(\frac{a^{2}-(z / c) b^{2}}{a}\right)}+\frac{y^{2}}{\left[b\left(1-\frac{z}{c}\right)\right]^{2}}=1 \equiv\left\{\begin{array}{l}
x=\left(a-\frac{z b^{2}}{a c}\right) \sin t \\
y=b\left(1-\frac{z}{c}\right) \cos t
\end{array}\right.
$$

where $z$ and $t$ are the two parameters of the previous expression, and the parameter $z$ stands for the height of the cross-section curves with respect to the plane of height zero, or plane where the generator ellipse is located. The parameter $t$ stands for the angle formed by the vertical plane holding the generating line for a constant value of $t$ with the vertical plane $x=0$. A better exposition for the parametric representation of a surface, is detailed in Appendix II.

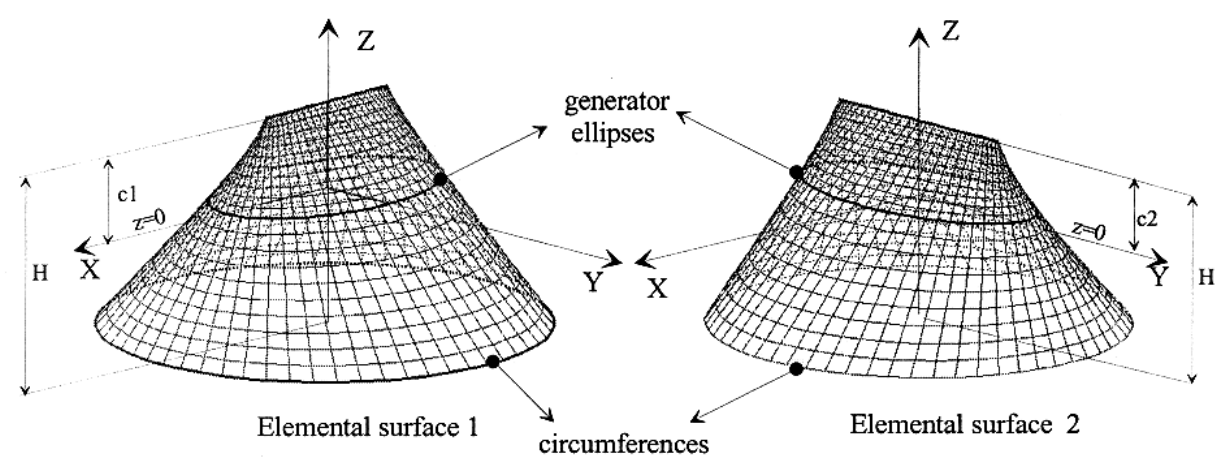

Figure 6

As was mentioned before, a surface that links the straight ridge to the circular base will have to be obtained, and it can easily be seen that such a surface comes about as the result of extracting it from the general one (see the encircled detail of Figure 5), thus obtaining the elemental surface that will be used for the development of the cathedral. To use our own terminology, starting with 
the general surface shown in Figure 5, called "elemental surface 1", and rotating it by $90^{\circ}$ we obtain "elemental surface 2", shown in Figure 6. The positioning of these figures is such that "elemental surface 1" will correspond to the surface having both the major axis of the generator ellipse and its straight straight ridge are parallel to the $x$-axis. Therefore, both the major axis of the generator ellipse and the straight ridge of "elemental surface 2" are parallel to the y-axis.

From what has been discussed up to this point, the project will consist of the interpenetration between the two surfaces rotated $90^{\circ}$ with respect to each other, or in othe words, all the crosssection or director ellipses of the two elemental surfaces cutting each other perpendicularly.

The following data are known:

- $\quad$ Radius $R$ of the base circle;

- Height $H$ between the ridge plane and the base one;

- Lengths $L_{1}$ and $L_{2}$ of the straight ridges of elemental surfaces " 1 " and " 2 " respectively.

The equation from which we begin the further mathematical development of the project is the given by equation (22) in its Cartesian form, where the linear relationship is given by $k=-z / c$, with $c$ being the distance between the generator ellipse located in the plane $z=0(k=0)$, and the straight ridge $(k=-1)$.

The next step consists in calculating the intersection between each pair of perpendicular director ellipses that are located in the same horizontal plane for every value of the parameter $Z$, yielding the four intersection points as solution of the equation system corresponding to the two elemental surfaces, in such a way that by achieving the same process for the entire set of the chosen values for the $z$ parameter, and further joining the homologous intersection points, we arrive at the four intersection curves.

The intent of the preceding considerations is to build three point arrays, for one to store the coordinates $(x, y, z)$ of the set of points belonging to the final surface, the rows of such arrays characterized by the $t$ parameter and the columns characterized by the $z$ parameter.

The quest for the values of the semiaxes $a$ and $b$ of the generator ellipse, and of the parameter $c$, in terms of the known data $R$ (base circle radius), $H$ (height between the ridge plane and the base one) and $L$ (length of the straight ridge), lead us to the results that were obtained in the previous section where we discussed how to deal with the Arrangement Law.

In expressions (23), the values $A$ and $B$ refer to the values of the semi-major and semi-minor axes of the ellipse (in this case to the radius of the base circle) located with respect to the plane of the straight ridge at a distance $\mathrm{H}, \mathrm{L}$ being the length of the straight ridge. 


$$
\begin{aligned}
& a=\frac{B^{2} \frac{L}{2}}{B^{2}-\left(A-\frac{L}{2}\right)^{2}} \\
& b=\frac{B\left(a-\frac{L}{2}\right)}{A-\frac{L}{2}} \\
& c=\frac{H}{\frac{A-a}{L}+1}
\end{aligned}
$$

The sections that follow consist of particularizing the aforementioned values of $a, b$ and $c$ for each of the two cathedral types in terms of whether the Latin Immisa cross or the Greek cross is chosen for the ridge plan, along with the description of the interpenetration process between the two elemental surfaces 1 and 2 in order to generate the eventual resultant surfaces for the designs. The project for each type of cross is illustrated.

\section{Project of a cathedral based on the Latin Immisa cross}

Defining the Latin Inmisa cross as that whose limbs are unequal and centered at a point located upon the vertical $z$-axis, we will designate as $L_{1}$ the length of the minor arm of the cross parallel to the $x$-axis and belonging to elemental surface 1. We will designate as $L_{2}$ the length of the major arm of the cross parallel to the $y$-axis and belonging to elemental surface 2 , such that the values of the semiaxes and the relative locations of every one of the elemental surfaces will be determined according to the expressions (23).

The expression for elemental surface 1 is deduced from equation (22) and given by:

$$
\left.\begin{array}{l}
\mathrm{x}=\left(\mathrm{a}_{1}-\frac{\mathrm{z} \mathrm{b}_{1}^{2}}{\mathrm{a}_{1} \mathrm{c}_{1}}\right) \sin \mathrm{t}_{1}=A_{1} \sin t_{1} \\
\mathrm{y}=\mathrm{b}_{1}\left(1-\frac{\mathrm{z}}{\mathrm{c}_{1}}\right) \cos \mathrm{t}_{1}=\mathrm{B}_{1} \cos \mathrm{t}_{1}
\end{array}\right\} \quad \begin{aligned}
& A_{1}=\left(\mathrm{a}_{1}-\frac{\mathrm{z} \mathrm{b}_{1}^{2}}{\mathrm{a}_{1} \mathrm{c}_{1}}\right) \\
& B_{1}=\mathrm{b}_{1}\left(1-\frac{\mathrm{z}}{\mathrm{c}_{1}}\right)
\end{aligned}
$$

The expression for elemental surface 2 is deduced from the equation (22) by a rotation of $90^{\circ}$, and is given by: 


$$
\begin{aligned}
& \left.x=b_{1}\left(1-\frac{z}{c_{1}}\right) \sin t=B_{2} \sin t_{2}\right) \quad B_{1}=b_{1}\left(1-\frac{z}{c_{1}}\right) \\
& \left.y=\left(a_{2}+\frac{\left(c_{1}-c_{2}-z\right) b_{2}{ }^{2}}{c_{2} a_{2}}\right)\right\} A_{2}=\left(a_{2}+\frac{\left(c_{1}-c_{2}-z\right) b_{2}{ }^{2}}{c_{2} a_{2}}\right)
\end{aligned}
$$

Additionally, we want to take into account the following relationships:

$$
\left.\begin{array}{l}
A_{1}=a_{1}\left(1-\frac{z}{c_{1}}\left(\frac{b_{1}^{2}}{a_{1}^{2}}\right)\right) \\
\mathrm{B}_{0}=B_{1}=B_{2}=b_{1}\left(1-\frac{z}{c_{1}}\right) \\
A_{2}=a_{2}\left(1+\frac{\left(c_{1}-c_{2}-z\right)}{c_{2}}\left(\frac{b_{2}^{2}}{a_{2}^{2}}\right)\right)
\end{array}\right\}
$$

where the values $a_{1}, b_{1}, c_{1}$ and $a_{2}, b_{2}, c_{2}$, for elemental surfaces 1 and 2 respectively, are given by the following expressions obtained from (23) by simply making $A=B=R$ and identifying $L$ by $L_{1}$ and $L_{2}$, depending on the elemental surface to which it refers.

$$
\begin{aligned}
& a_{1}=\frac{2 R^{2}}{4 R-L_{1}} ; \quad b_{1}=\frac{R\left(2 R-L_{1}\right)}{4 R-L_{1}} ; \quad c_{1}=\frac{H b_{1}}{R}=\frac{H\left(2 R-L_{1}\right)}{4 R-L_{1}} \\
& a_{2}=\frac{2 R^{2}}{4 R-L_{2}} ; \quad b_{2}=\frac{R\left(2 R-L_{2}\right)}{4 R-L_{2}} ; \quad c_{2}=\frac{H b_{2}}{R}=\frac{H\left(2 R-L_{2}\right)}{4 R-L_{2}}
\end{aligned}
$$

It is interesting to mention as well, as can be proved by making the calculations, that:

$$
\frac{c_{1}}{b_{1}}=\frac{c_{2}}{b_{2}}=\frac{H}{R},
$$

which justifies the fact that yields to $B_{1}=B_{2}=B_{0}$.

Eventually it can be verified that the sum of the semiaxes $a_{1}$ and $b_{1}$, as well as $a_{2}$ and $b_{2}$ from the generator ellipses correspond to the values of the radius $R$ of the base circumference, which is a given datum.

We want to emphasize the fact that the generator ellipses of the elemental surfaces does not lie in the same plane (Figure 7), hence the mathematical expression for one of the two surfaces will 
have to be referred to in terms of the other. Therefore the values of the parameter $z$ of the second surface with respect to the plane $z=0$ of the generator ellipse of the first surface, according to $Z^{\prime}=$ $z-\left(c_{1}-c_{2}\right)$, a relationship that appears explicity in the value $A_{2}$.

When the system made up of equations (24) and (25) is resolved, the result yields the loci of the space points common to the two surfaces from which the eventual cathedral is formed, loci that will also be the outcome of the interpenetration process.

In consequence of what has been discussed above, the equations that define the nonplanar curves that result from the process of interpenetration for the case of Latin Inmisa cross are given thus:

$$
\left\{\begin{array}{l}
x=\mu(z)= \pm A_{1} B_{0} \sqrt{\frac{B_{0}{ }^{2}-A_{2}^{2}}{B_{0}{ }^{4}-A_{1}^{2} A_{2}^{2}}} \\
y=\sigma(z)= \pm A_{2} B_{0} \sqrt{\frac{B_{0}^{2}-A_{1}^{2}}{B_{0}{ }^{4}-A_{1}^{2} A_{2}^{2}}}
\end{array}\right.
$$

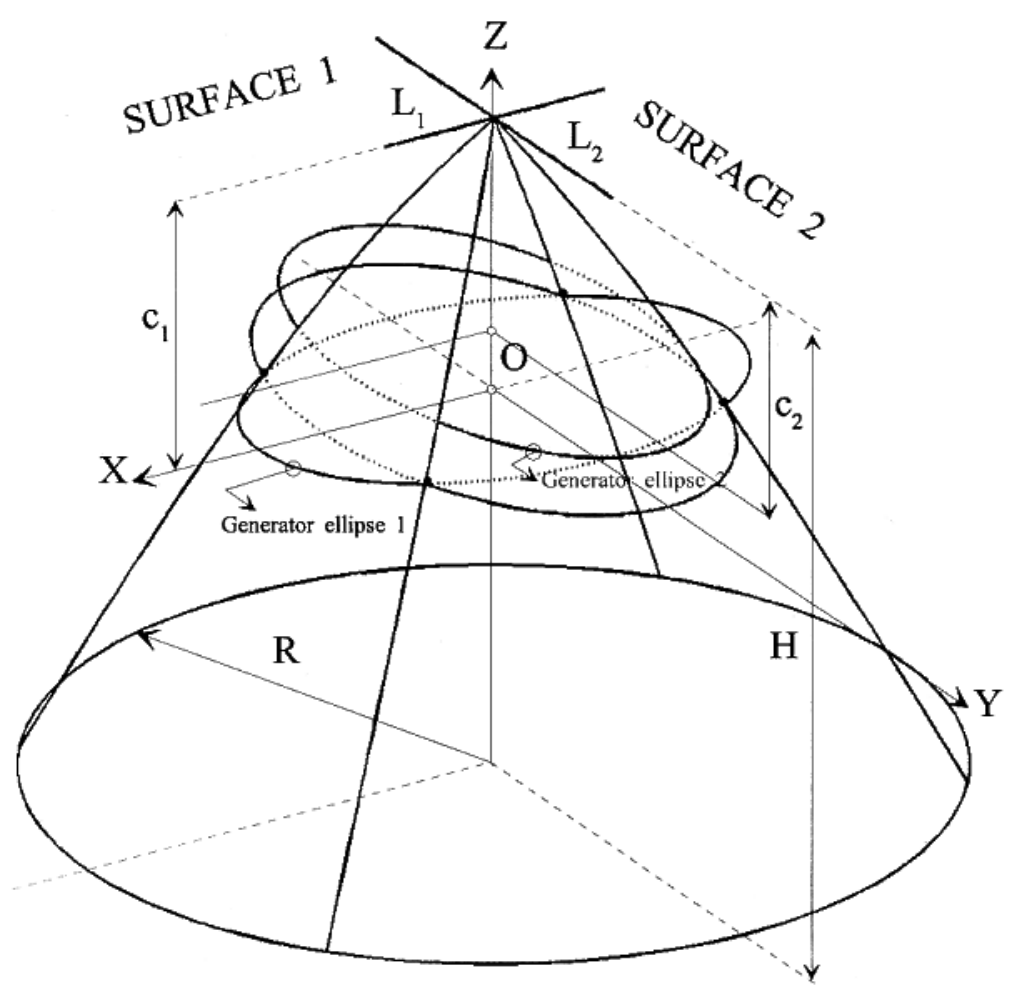

Figure 7

The process of interpenetration consists, then, of first obtaining the four possible points of intersection generated by each of the ellipses belonging to elemental surfaces 1 and 2 situated on 


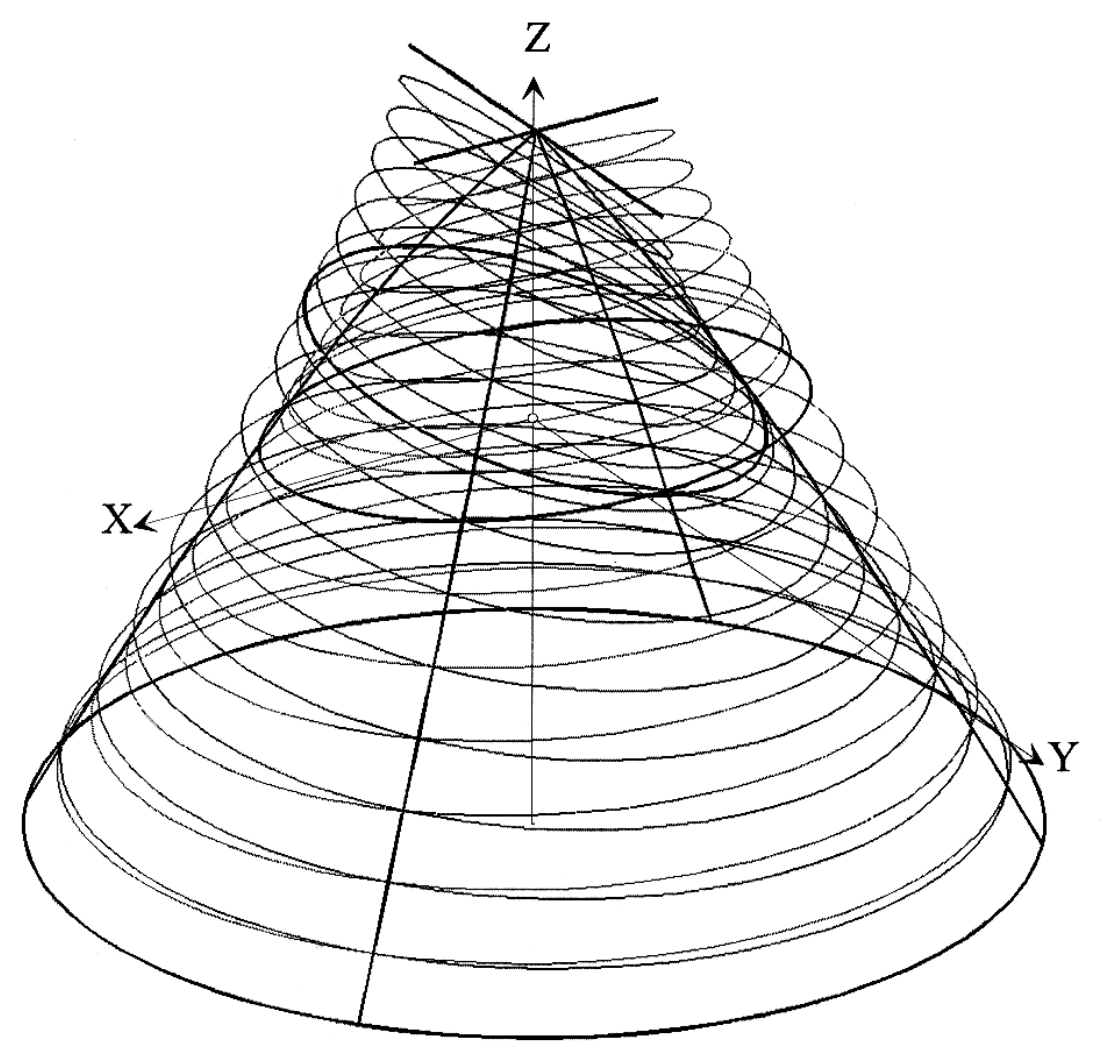

Figure 8

the same plane defined by parameter $z$. The next step is to eliminate the zones or stretches of the direction curves that are concealed (Figures 7 and 8 ). This gives the utilizable encircling surface of the final resultant surface. The meeting of all homologous points of intersection will define the four intersecting curves between the two elemental surfaces (Figures 7, 8, 9).

For the next phase, the starting point will be the previous calculation process, which consisted of selecting a determined number of direction curves that are common to the two surfaces of the cathedral, calculating their respective dimensions with reference to the plane containing the generating curve and which will be determined by parameter $z$. This will also supply the height of each direction plane with respect to the plane of the base circumference, the value of which is given by $h=H-c+z$. Then a suitable and sufficient number of generating straight lines is determined using parameter $t$, defined as the angle formed by the vertical plane that contains the direction straight line with the plane $x=0$. Values of $t$ in the direction of the $Y$ axis towards the $X$ axis are taken as positive, that is to say, clockwise in a Cartesian reference system. 


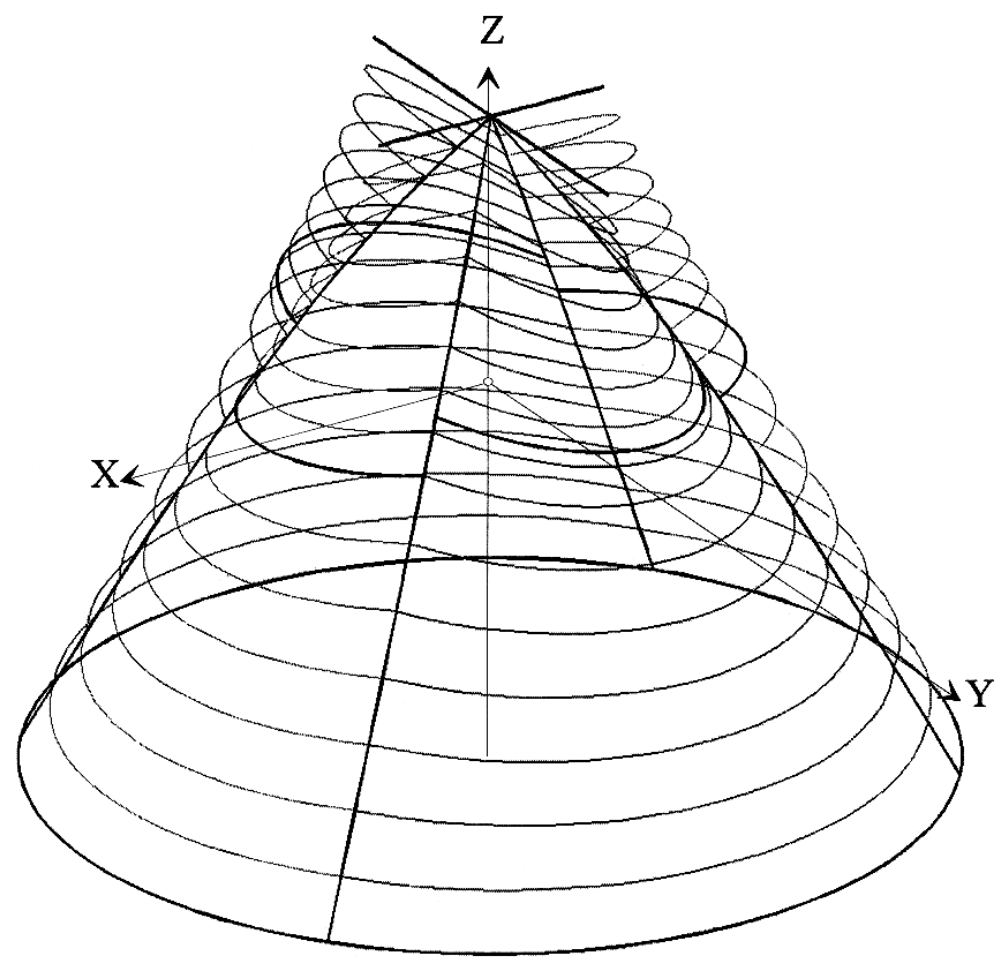

Figure 9

The next step is to determine all the points at which each previously selected generating straight line cuts each and every one of the direction curves also selected in accordance with what was discussed in the paragraph above. Having obtained all the points of intersection, a point matrix is created for each of the $x, y, z$ coordinates defined as $[X],[Y]$ and $[Z]$. The columns for all the previous matrices will reflect the order number corresponding to the direction plane, beginning with the ridge plane and moving as far as the base plane. The rows, on the other hand, represent the order number of the generating straight lines beginning with the value $t=0$ and finishing with $t=2 \pi$ in a clockwise direction. Hence each place within the corresponding matrix defined by its row and column will correspond to the value of the $x$ coordinate for the $[X]$ matrix, $y$ for $[Y]$ and $z$ for the corresponding $[Z]$ matrix. From all the points obtained above, those which are inside the resultant surface will have to be disregarded using the process of interpenetration. It is advisable for the previously selected generators to include those that pass through the points of intersection of each pair of direction ellipses situated in the planes whose $z$ parameter values were also selected.

From the point of view of construction, this cathedral project could be accomplished in two ways. One would involve the technique of reinforced concrete shells [Candela 1985; Faber 1970] for spans that are not too large, while the other would involve the use of metal structures for large spans. In either of these methods, one important feature is the absence of intermediate pillars, 

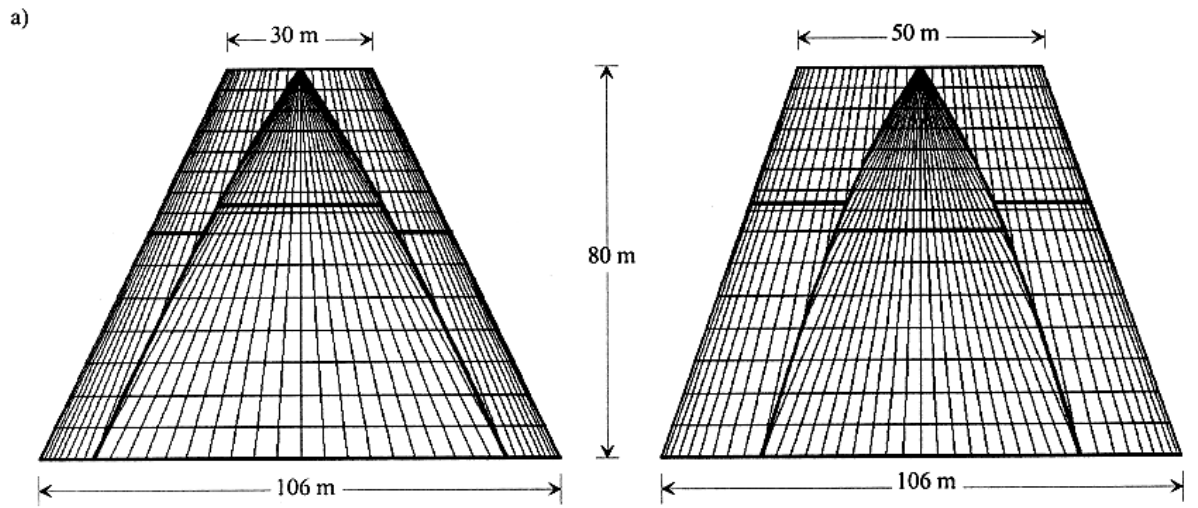

b)
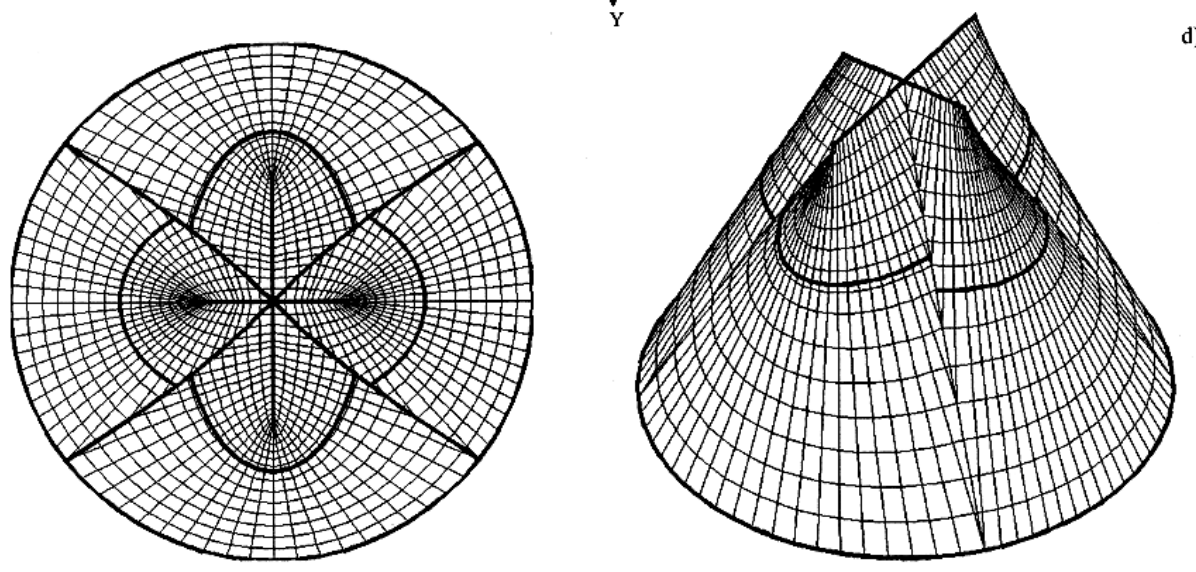

Figure 10 


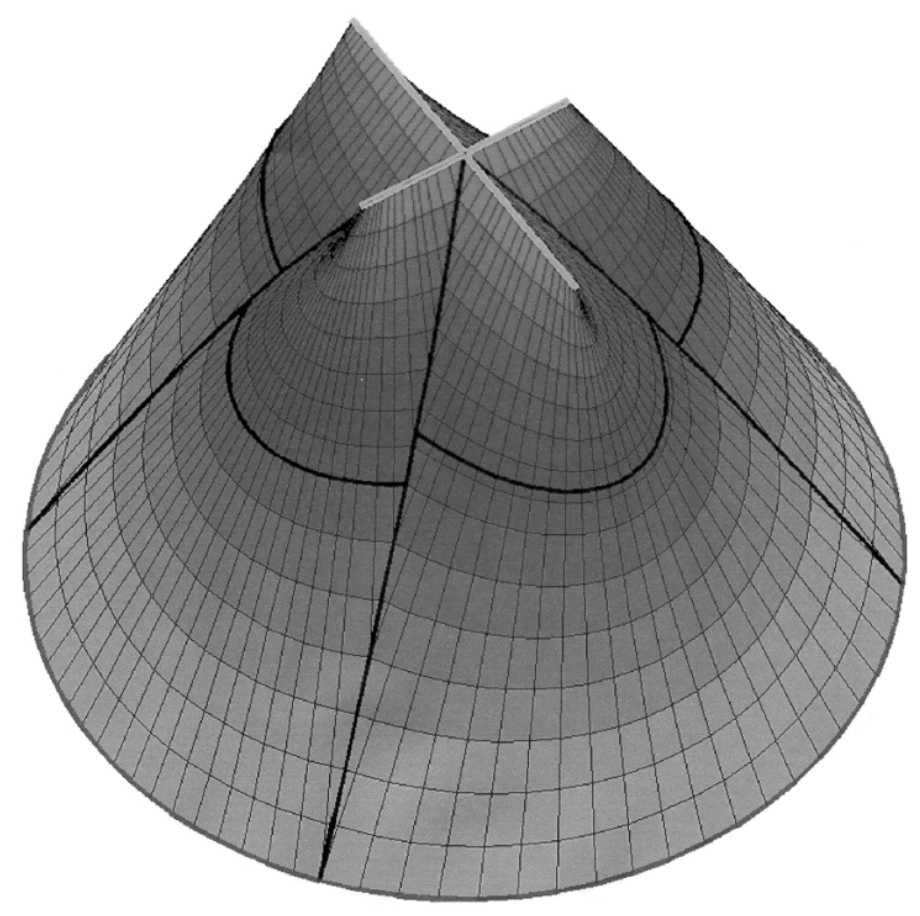

Figure 11

allowing completely open and diaphanous spaces. In those structures where the use of laminar reinforced concrete structures involves high building costs due in part to an excessive increase in thickness of the concrete layers, and where membrane stress calculations are no longer reliable for this type of structure, it is preferable to resort to metal structures involving the use of stronger, lighter and more resistant steels making it possible to achieve spans of up to 300 meters without intermediate supports.

Having obtained the point matrices, in the construction process using the technique of reinforced concrete laminar structures, the phase of erecting the wooden formwork would involve raising wooden pillars of length $h$ for each point $Q(x, y, z)$ projected on the base circumference plane. The coordinates are stored in the corresponding matrices mentioned above, allowing the assembly of wooden strips or boards in pillar form. The ends of the pillars are subsequently joined, giving rise to the generating straight lines such that in certain sections of the cathedral they will go from the straight ridge to the base circumference, and in others from the straight ridge to the corresponding point on the curve of intersection, between the two surfaces, thereby forming the cathedral surface. After the formwork, the necessary reinforcing bars are positioned and the concrete poured in to form the reinforced concrete. Once the concrete has set, the formwork is removed, revealing a curved concrete shell of a certain thickness capable of providing adequate rigidity and strength to withstand and absorb the stresses generated. The final surface 


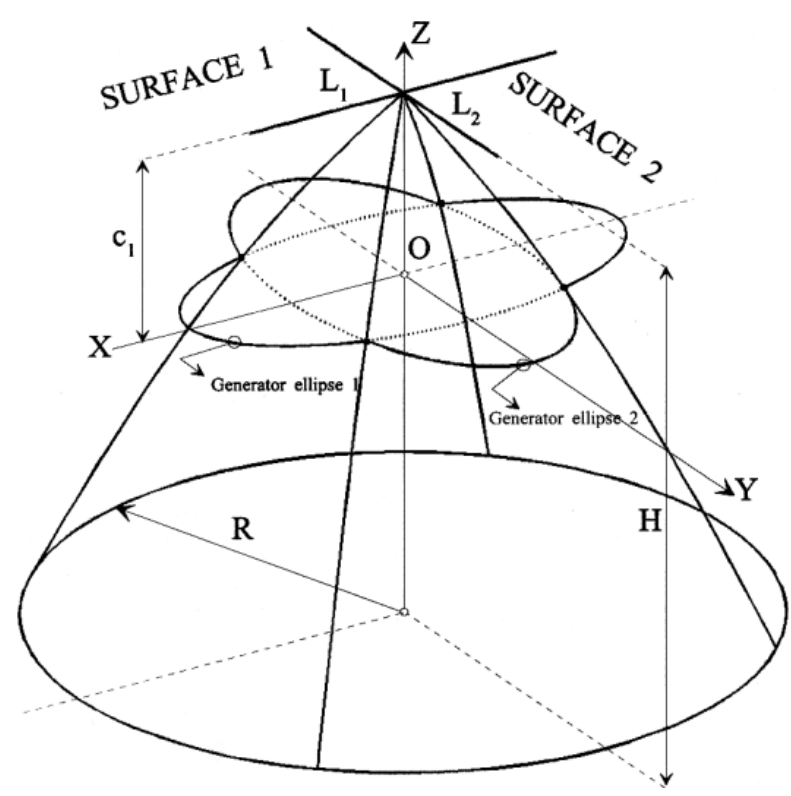

Figure 12

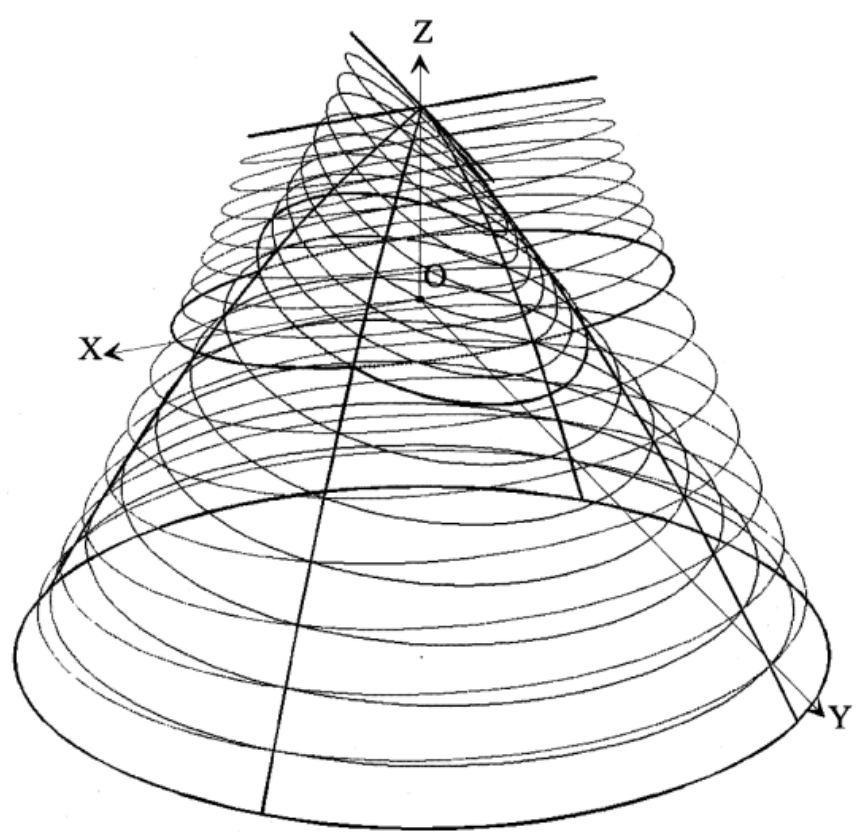

Figure 13

NEXUS NETWORK JOURNAL - VOL. 3, NO. 1, 200163 


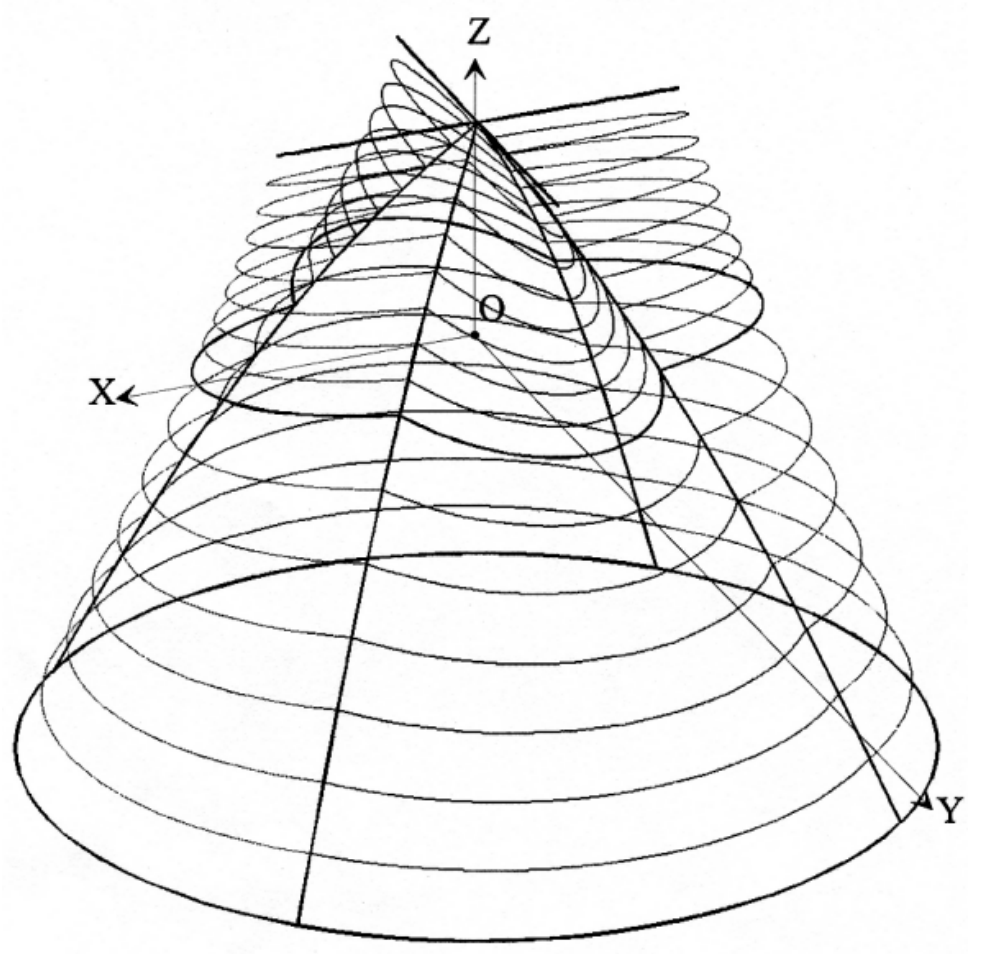

Figure 14

will create a totally open interior space with no internal columns to take away light and space, one one of the merits of this type of construction, devised by Spanish architect Félix Candela [Candela 1985; Faber 1970].

Designed with care, these surfaces would allow the creation of openings to accommodate rose windows and other large apertures to let light enter. Glazing these openings with suitably colored glass, in conjunction with design of the top part of the space as a transparent cross would project an extraordinary halo of light onto the altar and inside the cathedral.

The result of the cathedral project with the Latin Immisa cross is shown in Figure 10 and Figure 11, which takes account of the data $R=53$ metres, $H=80$ metres (which corresponds to the main dimensions of the actual Río de Janeiro cathedral), as well as the arms of the cross with values of $L_{1}=40$ meters and $L_{2}=50$ meters ("golden" proportions).

\section{Project for the cathedral based on the Greek cross}

The Greek cross is a particular case of the Latin Immisa cross where the arms have the same length equal to $L$. The corresponding expressions given in (27) now become those given in (29). It can be easily verified, as with the Latin Immisa cross, that the sum of the semiaxis $a$ and $b$ of the generator ellipse corresponds to the radius $R$ of the base circumference which is given.

64 NEXUS NETWORK JOURNAL - VOL. 3, NO. 1, 2001 


$$
\begin{aligned}
& \mathrm{a}_{1}=\mathrm{a}_{2}=\mathrm{a}=\frac{2 \mathrm{R}^{2}}{4 \mathrm{R}-\mathrm{L}} \\
& \mathrm{b}_{1}=\mathrm{b}_{2}=\mathrm{b}=\frac{\mathrm{R}(2 \mathrm{R}-\mathrm{L})}{4 \mathrm{R}-\mathrm{L}} \\
& \mathrm{c}_{1}=\mathrm{c}_{2}=\mathrm{c}=\frac{\mathrm{H}}{\mathrm{k}+1}=\frac{\mathrm{Hb}}{\mathrm{R}}=\frac{\mathrm{H}(2 \mathrm{R}-\mathrm{L})}{4 \mathrm{R}-\mathrm{L}}
\end{aligned}
$$

In the discussion up to this point, we have worked out the values of $a, b, c$ according to the equations of the previous case, since the Greek cross is a particular case of the Latin Immisa cross. The corresponding equations will be as follows:

For elemental surface 1, the straight ridge of which lies parallel to the $X$ axis, we have:

$$
\left.\begin{array}{l}
x=\left(a-\frac{z b^{2}}{a c}\right) \sin t_{1}=A_{1} \sin t_{1} \\
y=\left(1-\frac{z}{c}\right) \cos t_{1}=B_{1} \cos t_{1}
\end{array}\right\} \begin{aligned}
& A_{1}=\left(a-\frac{z b^{2}}{a c}\right) \\
& B_{1}=b\left(1-\frac{z}{c}\right)
\end{aligned}
$$

For elemental surface 2, the straight ridge of which lies parallel to the $Y$ axis, and turned $90^{\circ}$ with respect to elemental surface 1, we have:

$$
\left.\begin{array}{l}
x=b\left(1-\frac{z}{c}\right) \sin t_{2}=B_{2} \sin t_{2} \\
y=\left(a-\frac{z b^{2}}{a c}\right) \cos t_{2}=A_{2} \cos t_{2}
\end{array}\right\} \begin{aligned}
& B_{2}=B_{1} \\
& A_{2}=A_{1}
\end{aligned}
$$

In the previous expressions it can be observed that $A_{1}=A_{2}=A_{0}$ and $B_{1}=B_{2}=B_{0}$, these coefficients dependent on the parameter $z$. Thus, the system formed with the four previous equations will lead to the loci of points belonging to the plane curves in accordance with the solution (32) shown below, such that the intersection of such plane curves by whatever plane perpendicular to the $Z$ axis gives rise to the four common points of the two ellipses located in the same cutting plane. 

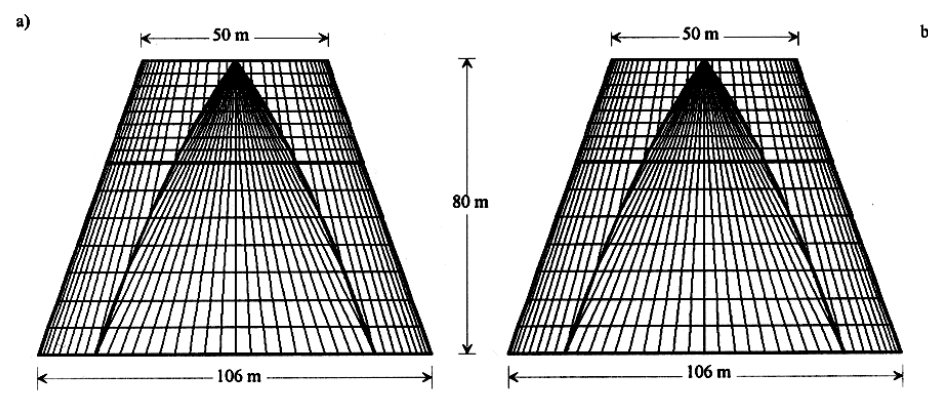

c)
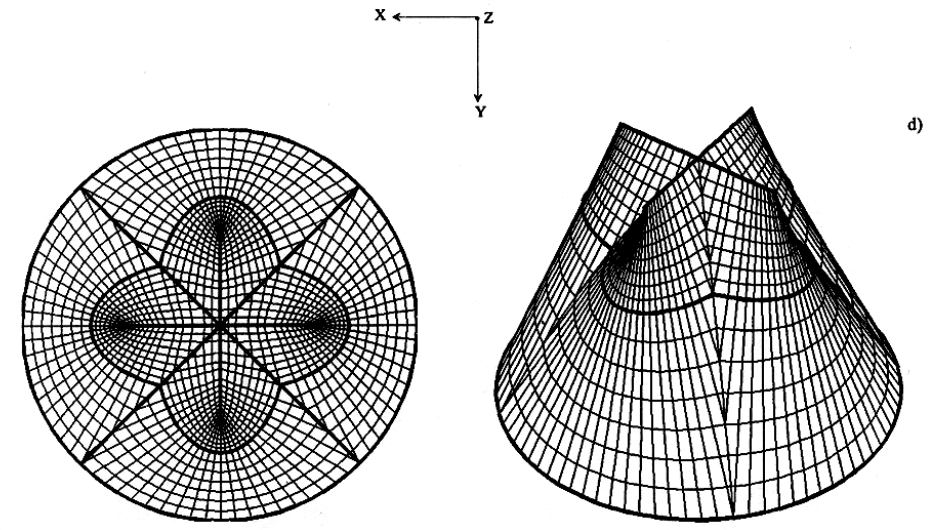

Figure 15

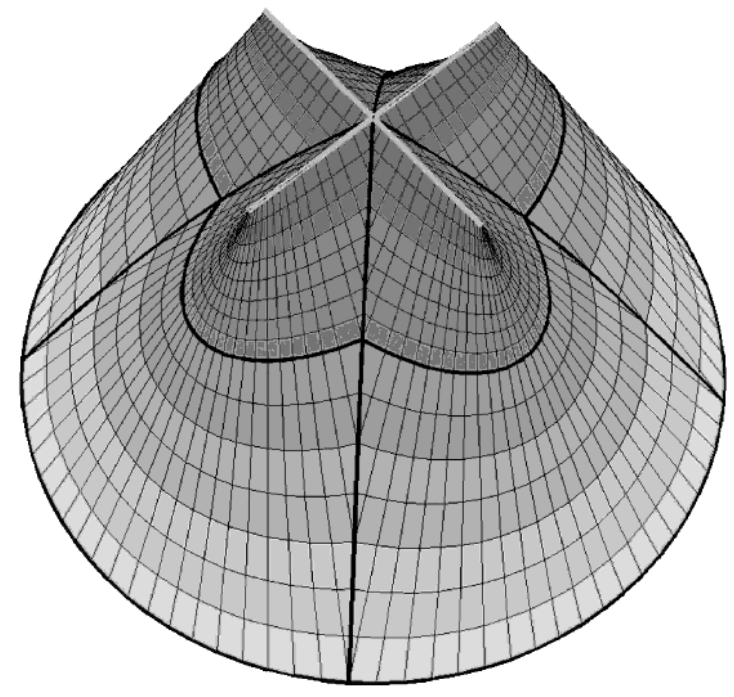

Figure 16

66 NEXUS NETWORK JOURNAL - VOL. 3, NO. 1, 2001 


$$
\left.\begin{array}{l}
x= \pm \frac{A_{0} B_{0}}{\sqrt{A_{0}^{2}+B_{0}^{2}}} \\
y= \pm \frac{A_{0} B_{0}}{\sqrt{A_{0}^{2}+B_{0}^{2}}}
\end{array}\right\} \equiv x= \pm y=\frac{a b\left(1-\frac{z}{c}\left(\frac{b^{2}}{a^{2}}\right)\right)\left(1-\frac{z}{c}\right)}{\sqrt{a^{2}\left(1-\frac{z}{c}\left(\frac{b^{2}}{a^{2}}\right)\right)^{2}+b^{2}\left(1-\frac{z}{c}\right)^{2}}}
$$

In Figures 12, 13 and 14, the process of interpenetration discussed in the previous sections is illustrated schematically for the Greek cross, showing the parallel planes of the base and of the straight ridge, between which the surface is formed, as well as the planes belonging to elemental surfaces 1 and 2 . In this case the two generator ellipses lie in the same plane, since the arms of the cross are of the same length as well as the heights of $c_{1}$ and $c_{2}$ from each respective arm to the generator ellipse plane $(z=0)$. These figures also illustrate the four intersection points generated by the two generator ellipses, through which the four intersection curves pass, as result of the process of interpenetration between the two surfaces.

The final result of the Greek cross project is illustrated in Figures 15 and 16, where the given parameters were again $R=53$ meters and $H=80$, with the length of the arms of the cross $L_{1}=L_{2}=30$ metres.

\section{The architectural conclusion}

In addition to the high level of originality of these design projects from a structural and mathematical point of view, demonstrated in the fact that the light generated in the ridge plane is an integral part while the straight ridge itself forms the roof and support, the symbolic religious character suggested by these surfaces is unquestionable and worthy of note. This religious character is expressed by the way the upper level in the form of a cross seems to embrace the whole space enclosed by the surface of the cathedral developed here, thus aspiring to join together the upper, divine, level, with the lower, terrestrial, one.

\section{Appendix I. Definition of the conic ellipse}

The ellipse as a conic section can be considered as generated by the cutting of a cone by a plane that cuts all its generating lines, and it is therefore defined as the loci of points of the plane whose sum of distances between two points is constant.

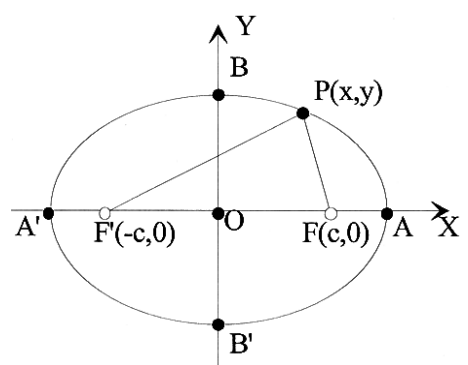

Figure I.1 
Taking into account the ellipse illustrated in the Figure I.1, the following points are defined.

- The fixed points $\mathrm{F}, \mathrm{F}^{\prime}$ are called the foci of the ellipse;

- The distance $\overline{F F^{\prime}}=2 c$ is called focal length;

- The distances $\overline{F^{\prime} P}$ and $\overline{F P}$ are called radius vectors;

- The sum $\overline{P F}+\overline{P F^{\prime}}=2 a$ is a constant value, as it can be demonstrated according to the definition of the ellipse itself;

- The difference $a^{2}-c^{2}$ will be called $b^{2}$ and will be positive, since $a^{2}>c^{2}$;

- The line segment distance $\overline{A A^{\prime}}=2 a$ will be called the major axis of the ellipse;

- The line segment distance $\overline{B B^{\prime}}=2 b$ will be called the minor axis of the ellipse.

Taking line segment $\overline{F F^{\prime}}$ as the horizontal axis and the perpendicular to the point in the middle of the line segment $\overline{F F^{\prime}}$ as the vertical axis, and from the definition of the ellipse given above, it comes to:

$$
\overline{P F}+\overline{P F^{\prime}}=2 a \Rightarrow \sqrt{(\mathrm{x}-\mathrm{c})^{2}+y^{2}}+\sqrt{(\mathrm{x}+\mathrm{c})^{2}+y^{2}}=2 a
$$

By operating upon that equation and taking into account the relation $a^{2}-c^{2}=b^{2}$, the following expression is obtained for the canonical equation of the ellipse:

$$
\frac{x^{2}}{a^{2}}+\frac{y^{2}}{b^{2}}=1
$$

\section{Appendix II. Surfaces and curves on a surface [Granero Rodriguez 1985]}

In the affine three-space, the term "curve" applies to the entire collection $C \subset \mathfrak{R}^{3}$ of three coordinates $(x(t), y(t), z(t))$, such that $x(t), y(t), z(t)$ are continuous functions at a certain interval I. Hence, the equations:

$$
\left\{\begin{array}{l}
x=x(t) \\
y=y(t) \\
z=z(t)
\end{array}\right.
$$

are called the parametric equations of the curve $\mathrm{C}$ (tridimensional line).

Considering the existing isomorphism between the free vectors of space and the points of $\mathrm{E}_{3}$, it is often more suitable to define the curve C by a free vector $\vec{v}(t)$ of components $(x(t), y(t)$, $z(t))$. In this case it is clear that by changing the parameter $t$, the vector end will draw the curve C (Figure II.1). 


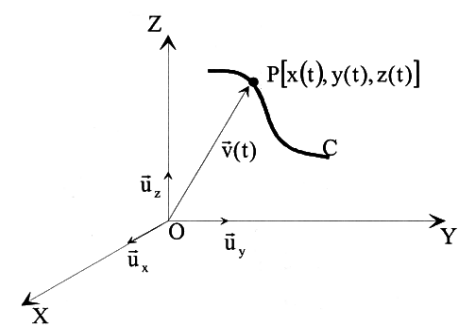

Figure II.1

The equation $\vec{v}(t)=x(t) \vec{u}_{x}+y(t) \vec{u}_{\mathrm{y}}+z(t) \vec{u}_{\mathrm{z}}$ is called the vectorial equation of $\mathrm{C}$.

When the curve is located in a plane is called a plane curve. Otherwise it is called a nonplane curve.

In the affine three-space the term "surface" applies to the entire collection of points $S \subset \mathfrak{R}^{3}$ of coordinates $(x(\lambda, \mu), y(\lambda, \mu), z(\lambda, \mu))$, such that $x(\lambda, \mu), y(\lambda, \mu), z(\lambda, \mu)$ are continuous functions in a certain domain $\mathrm{D} \subseteq \mathfrak{R}^{2}$.

The equations

$$
\left\{\begin{array}{l}
x=x(\lambda, \mu) \\
y=y(\lambda, \mu) \\
z=z(\lambda, \mu)
\end{array}\right.
$$

are called parametric equations of the surface $S$.

In the same way, based on the isomorphism between free vectors and points, we would be able to define the surface $S$ by a free vector or position vector $\vec{v}(\lambda, \mu)$ of components $(x(\lambda, \mu), y(\lambda, \mu)$, $z(\lambda, \mu))$. In these conditions, by varying the parameters $\lambda$ and $\mu$, the position vector end will travel through the surface $S$ (Figure II.2).

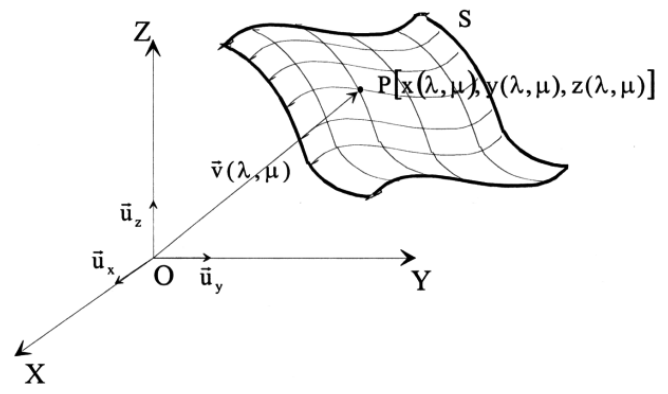

Figure II.2

The equation

$$
\vec{v}(\lambda, \mu)=x(\lambda, \mu) \vec{u}_{x}+y(\lambda, \mu) \vec{u}_{y}+z(\lambda, \mu) \vec{u}_{z}
$$


will be called the vectorial equation of such a surface. Often it is feasible to remove the two parameters $\lambda$ and $\mu$ among the three equations (II.2), giving rise to an equation of the form $\mathrm{F}(x, y, z)=0$, or $z=\mathrm{f}(x, y)$, which is called the Cartesian equation of the surface. Reciprocally if the surface is defined by $z=\mathrm{f}(x, y)$, making $x=\lambda, y=\mu$ yields the following parametric equations

$$
\left\{\begin{array}{l}
x=\lambda \\
y=\mu \\
z=f(\lambda, \mu)
\end{array}\right.
$$

Likewise, let us notice that if whatever relationship, as for instance $\lambda=f(\mu)$, between both parameters is established, the position vector $\vec{v}(\lambda, \mu)=\vec{v}[f(\mu), \mu]$ will depend solely on one of those parameters, and consequentely, a curve upon the surface will be drawn. Again, from what has preceded, the result will be that when one of the two parameters is fixed, or when these are under the relationship $\lambda=f(\mu)$, or $\left\{\begin{array}{l}\lambda=\lambda(t) \\ \mu=\mu(t)\end{array}\right.$, we will have a curve on the surface.

Example:

Given the surface $S$ defined in parametrics as:

$$
S \equiv\left\{\begin{array}{l}
x=\lambda+\mu \\
y=\lambda-\mu+4 \\
z=-2\left(\lambda^{2}+\mu^{2}+4 \lambda-4 \mu\right)
\end{array}\right.
$$

by isolating $\lambda$ and $\mu$ in terms of $x$ and $y$ between the two first equations, which are linear, by substituting in the third we will have $z=\mathrm{f}(x, y)$. However in this case we can very easily obtain $z=$ $\mathrm{f}(x, y)$ by just noticing that:

$$
x^{2}+y^{2}=\lambda^{2}+\mu^{2}+2 \lambda \mu+\lambda^{2}+\mu^{2}+16-2 \lambda \mu+8 \lambda-8 \mu=2\left(\lambda^{2}+\mu^{2}+4 \lambda-4 \mu\right)+16,
$$

where

$$
-2\left(\lambda^{2}+\mu^{2}+4 \lambda-4 \mu\right)=16-x^{2}-y^{2} .
$$

Therefore, $z=16-x^{2}-y^{2}$ or $x^{2}+y^{2}+z-16=0$ represents the Cartesian equation of the surface.

It is very simple to display the preceding surface, since all its intersection through planes $z=k$ parallel to the horizontal are circumferences with radii that become uniformly shorter until becoming null at point $(0,0,16)$.

Likewise, let us notice that:

$$
S \equiv\left\{\begin{array}{l}
x=\lambda \\
y=\mu \\
z=16-\lambda^{2}-\mu^{2}
\end{array}\right.
$$

70 NEXUS NETWORK JOURNAL - VOL. 3, NO. 1, 2001 
are less difficult parametric equations of this surface than the preceding ones.

Appendix III. Ruled surfaces [Granero Rodriguez 1985].

It is well known from analytic geometry that every straight line in space can be expressed in the form:

$$
\left.\begin{array}{l}
x=m z+a \\
y=n z+b
\end{array}\right\}
$$

where $m, n, a, b \in \Re$.

It is clear that when the coefficients $m, n, a, b$ are fixed, the system (III.1) will represent a sole straight line in space; in other words, to every quadruple set of $(m, n, a, b) \in \mathfrak{R}^{4}$ will correspond a sole straight line of such space. If we make these four coefficients be in terms of a parameter $\zeta \in$ $\Re$, then for every value of $\zeta$ we will have a well-defined straight line such that by varying this parameter the result will be a loci of straight lines, that is, in a surface made up of straight lines.

A ruled surface is that which is generated by a straight line (generating line) which moves according to a given mathematical law.

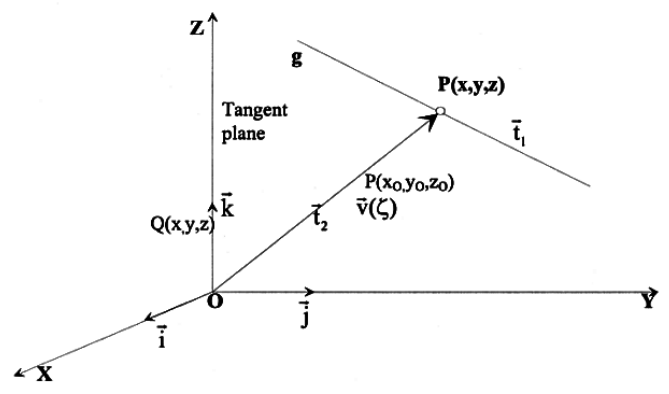

Figure III.1

Let be (III.1) a variable straight line whose coefficients $m, n, a, b$ depend on a parameter $\zeta$. In these conditions we will have the equations:

$$
\left.\begin{array}{l}
x=m(\zeta) \cdot z+a(\zeta) \\
y=n(\zeta) \cdot z+b(\zeta)
\end{array}\right\}
$$

representing the parametric equations of a surface (two equations with a sole parameter whose isolation will give raise to the Cartesian form). Since the vectorial equation of the surface is defined by means of the position vector of a given point $\mathrm{P}(x, y, z)$ (Figure III.1), we have:

$$
\vec{v}(\zeta)=x \overrightarrow{\mathrm{i}}+\mathrm{y} \overrightarrow{\mathrm{j}}+\mathrm{z} \overrightarrow{\mathrm{k}}
$$

where:

$$
\vec{v}(\zeta)=[\mathrm{m}(\zeta) \mathrm{z}+\mathrm{a}(\zeta)] \overrightarrow{\mathrm{i}}+[\mathrm{n}(\zeta) \mathrm{z}+\mathrm{b}(\zeta)] \overrightarrow{\mathrm{j}}+\mathrm{z} \overrightarrow{\mathrm{k}}
$$

will be its vectorial equation. Hence we will be able to say: 


$$
\left.\begin{array}{l}
x=m(\zeta) \cdot z+a(\zeta) \\
y=n(\zeta) \cdot z+b(\zeta) \\
z=z
\end{array}\right\}
$$

are as well the parametric equations of that surface. Let us take notice that last equation of the preceding system can be verified by every point of space and in consequence $z=Z$ will be all $\mathfrak{R}^{3}$.

It is clear that parametric equations (III.2) and (III.3) represent the same surface, since the system (III.3) can be considered as intersection of the surface defined as the system (III,2) with $Z=Z$, that is, with $\mathfrak{R}^{3}$. Furthermore, taking into account that Appendix II demonstrated the parametric expression of the surfaces through three equations with two parameters $\lambda$ and $\mu$, in this paper we have choosen the system (III.3) to achieve it, and by convenience $t$ and $z$ have been selected as parameters.

First published in the NNJ online Spring 2001

\section{References}

CANDELA, Félix. 1985. En defensa del formalismo y otros escritos. Bilbao: Ediciones Xarait.

FABER, Colin. 1970. Las estructuras de Candela. Mexico: CECSA.

GranERO-RodríGUEZ, Francisco. 1985. Álgebra y geometría analítica. Madrid and Mexico City: McGrawHill.

MarTín-Zorraquino, Juan V., Francisco Granero-Rodríguez, José Luis Cano-Martín, J.J. Doria-Iriarte, J.J. 1999. A Novel Version of the Cathedral Inspired in the Already Built one in Rio de Janeiro. Pp. 357364 in ISAMA 99. Nathaniel Friedman and Javier Barallo, eds. Proceedings of the International Society of the Arts, Mathematics and Architecture (ISAMA) 99 conference, 7-11 June 1999, San Sebastián.

MARTÍN-ZorRaQUinO, J.V.. Francisco Granero-Rodríguez, J.J.Doria-Iriarte, J.J. 1997. "Otras Aportaciones y Resultados sobre la Nueva Familia de Figuras Geométricas Elementales con Cónicas". 9º International Congress of Graphic Engineering. Bilbao - S. Sebastián: UPV-EHU.

Martín-Zorraquino, J.V., Francisco Granero-Rodríguez, J.J. Doria-Iriarte. 1998. "New Properties of Conic Sections and the Ruled Surfaces Deriving from them. Selected Applications in Architecture and Engineering". Pp. 431-438 in Mathematics \& Design 98. J. Barrallo, ed. Proceedings of the Second International Conference of Mathematics \& Design 98. Sebastián: UPV-EHU.

Martín-Zorraquino, J.V., Francisco Granero-Rodríguez and J.J. Doria-Iriarte. 1993. "Superficies Tridimensionales de Secciones Cónicas." Patent number P9301662/1993.

\section{The Authors}

Prof. Dr. Martín Zorraquino is an industrial engineer and Professor in Thermic Engineering at the High Technical School of Engineering in Bilbao (Spain) holding his professor's chair since 1966. He has been director of the Heat Engines Department at the same university since 1987. During the last years, he has run ten research projects financed by the Basque Country University and the Basque Government itself. He has written several research articles

Dr. Granero Rodriguez is an industrial engineer and Lecturer in Applied Mathematics at the High Technical School of Engineering in Bilbao. He has written several research workings and several books on mathematics, five of them published by the publishing house McGraw-Hill.

Dr. Cano Martín is an industrial engineer. He works as an engineer for a large firm located in the Basque Country. 